\title{
Alternative relaying for cooperative multiple-access channels in wireless vehicular networks
}

\author{
Peng $\mathrm{Xu}^{1^{*}}$, Zhiguo Ding ${ }^{2}$, Xuchu Dai ${ }^{1}$, loannis Krikidis ${ }^{3}$ and Athanasios V Vasilakos ${ }^{4}$
}

\begin{abstract}
In this paper, a novel spectrally efficient half-duplex cooperative transmission protocol is proposed for cooperative multiple-access channels in wireless vehicular networks, where multiple sources (vehicles) deliver messages to a common destination (roadside base station or roadside infrastructure) with the help of multiple decode-and-forward relays (roadside stations). The basic idea is to apply superposition coding at each transmitter in order to achieve the full diversity gain, where the linear zero-forcing detection is used at each relay to combat inter-relay interference. Compared to existing uplink cooperative protocols, the proposed scheme can exploit the cooperation involving both the relays and the sources. An achievable diversity-multiplexing trade-off is developed for the proposed transmission protocol. Even with strong inter-relay interference which has been ignored by many existing works, the proposed scheme can still approach the optimal multiple-input single-output upper bound. Numerical results have also been provided to demonstrate the performance of the proposed protocol.
\end{abstract}

Keywords: Cooperative multiple access; Wireless vehicular network; Alternative relaying; Diversity-multiplexing trade-off

\section{Introduction}

\subsection{Background}

Recent researches have paid much attention to wireless vehicular ad hoc networks (VANETs) since they are important to intelligent transportation, environment monitoring, etc $[1,2]$. Unlike the cellular networks, there does not exist a powerful base station in a vehicular ad hoc network, so peer-to-peer transmission is relied on. Correspondingly, a key problem is how to design the routing protocols to efficiently forward the source messages to the destination. One potential method is to design cross-layer protocols for mobile vehicular ad hoc networks. For example, the work in [3] has shown that cooperative transmission, a low-cost and effective alternative to multiple-input multiple-output (MIMO) techniques to enhance the physical layer performance, can bring its physical layer benefits to the upper layer, such as the design of routing protocols.

\footnotetext{
*Correspondence: mxp484@mail.ustc.edu.cn

${ }^{1}$ Department of Electronic Engineering and Information Science, University of Science and Technology of China, Huangshan Road, P.O.Box No.4, 230027, Hefei, Anhui, China

Full list of author information is available at the end of the article
}

Motivated by these benefits of cooperative transmission, this paper aims to design a novel cooperative transmission scheme for the multiple access channel (MAC) in the wireless vehicular networks.

Exploring cooperation among the source nodes has been studied in [4,5] for the MACs. The work in [4] proposed a cooperative multiple-access (CMA) transmission protocol using amplify-and-forward (AF) strategy, and its decode-and-forward (DF) version has been proposed in [5] by using superposition (SP) coding at each source, where the idea of superposition coding was first proposed in [6] to increase the system capacity. Specifically, the key idea of such superimposed cooperative schemes is that each user will share parts of its power with other users and transmit a mixture of its own and others' information. The diversity-multiplexing trade-off (DMT) [7] achieved by these schemes can approach the optimal multipleinput single-output (MISO) upper bound. However, such schemes only exploit the cooperation among the sources and are therefore constrained by the number of sources.

\section{是 Springer}

(c) 2014 Xu et al: licensee Springer. This is an Open Access article distributed under the terms of the Creative Commons Attribution License (http://creativecommons.org/licenses/by/2.0), which permits unrestricted use, distribution, and reproduction in any medium, provided the original work is properly credited. 


\subsection{Related work}

To further improve the reception robustness, exploiting relays as an extra dimension has been recognized as a cost-effective way, since the number of idle users is always larger than the active ones in a typical wireless network. However, extra time slots may be consumed to repeat source messages in relay transmission. For example, due to the half-duplex constraint, there exists a loss of spectral efficiency for the classical orthogonal transmission schemes $[8,9]$. The standard non-orthogonal transmission strategies in $[4,10]$ can make some improvement and achieve the full multiplexing gain but still suffer a significant diversity loss for large multiplexing gains, if compared with the optimal MISO upper bound. To overcome limitation of the available diversity, a successive relaying concept which aims to physically reuse the relay-destination channels was studied in [11-14]. The basic idea is to arrange two or more relays to alternatively retransmit the source messages. To deal with the inter-relay interference, the inter-relay link was usually assumed to be either sufficiently strong or weak in these DF-relaying works. A cooperative transmission protocol named as shifted successive DF relaying (SSDFR) was proposed in [14], where maximum likelihood (ML) decoding is utilized at two multiple-antenna relays. In [15], another cooperative transmission protocol, called relay-reuse DF (RRDF), has been proposed to the scenario with a single source node, which yields furthermore improvement in comparison with [14] due to the linear zero-forcing (ZF) detection which can reduce the computational complexity.

For a general uplink scenario with multiple users and relays, it is still a challenging problem to integrate the cooperation among the sources and the cooperation from the relays. In $[16,17]$, each relay utilized superposition coding to assist each source. However, the developed upper bound of DMT in [16,17] can only be achieved with some assumptions on the conditions of inter-relay channels. The work in [18] considered a more general scenario and proposed a cooperative protocol, called relay-assisted CMA (R-CMA), which carefully schedules the multiple sources and relays' transmission, but the optimal MISO bound can only be approached for a large number of relays. More works that considered such multi-user multi-relay networks can be found in [19-21], where the assistance of relays has been well exploited. Generally, these works only use the assistance of relays without the cooperation between sources, which cannot fully exploit the capacity of the uplink system.

\subsection{Contribution}

This paper aims to design a novel cooperative transmission protocol for a CMA channel to integrate the cooperation from dedicated relays and the mutual cooperation between users.

Specially, we consider a cooperative multiple-access communication scenario in which multiple source nodes communicate with the common destination with multiple half-duplex relays. Such a communication pattern is an important building block in wireless VANETs. For example, some proposals of intelligent vehicle highway systems (IVHS) have introduced a variety of vehicle-to-vehicle and vehicle-to-roadside-infrastructure communications. In this case, cooperation can take place between vehicles or between vehicles and roadside stations. When some vehicles wish to send messages to a common roadside base-station, they can cooperate with each other and also obtain external assistances from more powerful roadside stations with multiple antennas.

The proposed cooperative transmission protocol is termed as alternative-relaying decode-and-forward (ARDF) throughout this paper. The main novelty is that a new cooperative multiple-access transmission protocol has been designed by carefully applying these low-cost tools (zero-forcing detection, antenna selection, superposition coding, repetition-coded decode-and-forward, etc.) and fully exploiting the potentials of relay and source cooperation. To the best of the authors' knowledge, no existing works can effectively achieve this full cooperation. The basic idea of the proposed protocol is to combine the superposition coding [6] with RRDF scheme [15]. By carefully arranging the transmission process and applying superposition coding at transmitters, each source can be assisted by all the other nodes, including relays and other sources; by utilizing the linear ZF decoding in terms of RRDF transmission scheme, the reuse of the relaydestination links can be achievable in the scenario with a general inter-relay interference. Hence, the potential of such a system can be fully exploited, and the full diversity gain can be achievable. Antenna selection is also applied at each relay to select the best antenna for transmitting, which can preserve the full diversity gain and save transmit power.

DMT is used to evaluate the spectral efficiency of the proposed CMA protocol, since it has been recognized as an effective information-theoretic criterion. The achievable DMT for the proposed ARDF protocol for the CMA channel is developed by characterizing the associated outage probabilities of outage events. From the derived result, we can show that the DMT of the proposed scheme can approach the optimal MISO upper bound when the length of one cooperative data frame is large. Compared to the previous CMA schemes in [4,5] and R-CMA in [18], the proposed cooperative scheme achieves a better DMT curve at most multiplexing gain ranges, especially when the frame length is large enough. Monte Carlo simulation results have also been presented to demonstrate the 
outage probability performance of the proposed transmission protocol.

Compared to the point-to-point communication in [15], we consider a multi-user case for the addressed MAC scenario in this paper. As a consequence, DMT for the MAC channel in [22] should be adopted, the informationtheoretic criterion, which is developed based on the capacity region of MAC [23]. Specifically, define $X_{\mathcal{S}}=$ $\left\{X_{i}: i \in \mathcal{S}\right\}$, and $X_{i}(Y)$ is the channel input (output) variable. For a instantaneous channel state information (CSI) which is known at the receiver, the capacity region of the $M$-user MAC can be written as following [23]:

$$
\begin{aligned}
\sum_{i \in \mathcal{S}} R_{i} & <I\left(X_{\mathcal{S}} ; Y \mid X_{\mathcal{S}^{c}}, \mathbf{H}=H\right) \text { for all } \\
\mathcal{S} & \subseteq\{1,2, \cdots, M\}
\end{aligned}
$$

for some product distribution $p_{1}\left(x_{1}\right) \cdots p_{M}\left(x_{M}\right)$, where $R_{i}$ the data rate of source $i(i=1, \cdots, M)$ and $\mathbf{H}$ is the corresponding source-destination channel matrix. As can be observed from the above equation, the capacity region of MAC is a set of many inequalities with different conditions, which makes the calculation of DMT much more difficult than the single-user scenario in [15]. These challenges also make the corresponding analysis more valuable.

This paper is organized as follows. Section 2 describes the proposed CMA-ARDF transmission protocol. Section 3 presents the details of the ARDF transmission process of the proposed scheme. Section 4 outlines the achievable DMT and the Monte Carlo simulation results for the proposed protocol. Section 5 offers concluding remarks. The proofs of the final DMT result are provided in the 'Appendix' section.

\section{Protocol description}

A cooperative multiple-access scenario is considered in this paper, where multiple sources wish to communicate a common destination under the help of relays. In particular, the addressed scenario consists of two single-antenna sources (i.e., vehicles, denoted as $\mathrm{S}_{1}$ and $\mathrm{S}_{2}$ ), two $M_{r^{-}}$ antenna $\left(M_{r} \geq 2\right)$ relays (i.e., fixed roadside stations, denoted as $R_{1}$ and $R_{2}$ ), and one common single-antenna destination (i.e., base station, denoted as D). The abstract system model and its application to the wireless vehicular networks are shown in Figures 1 and 2, respectively. All channels are assumed to be frequency-flat quasi-static Rayleigh fading. Such a Rayleigh fading assumption is valid in a practical vehicular network since it is very likely that there does not exist a line-of-sight (LOS) component between a pair of VANET transceivers due to their relatively low antenna height $[3,24,25]$. All nodes are constrained by the half-duplex assumption and use the same transmission power. Time division multiplexing access (TDMA) is utilized in this scenario for its simplicity.

Every antenna at each relay is marked with a distinct index number from 1 to $M_{r}$. As shown in Figure 1, the column vector $\mathbf{h}_{i, R j}$ denotes the channel vector between the $i$-th source and $\mathrm{R}_{j}$, where $i, j=1,2$, and $h_{S i}$ represent the source-destination channel. The channel vector between $\mathrm{R}_{j}$ and $\mathrm{D}$ is denoted as a column vector $\mathbf{g}_{j}=$ $\left\{g_{j, 1}, \cdots, g_{j, M_{r}}\right\}$. Moreover, the column vector $\mathbf{h}_{j, m}$ denotes the inter-relay channel vector between the $m$-th antenna of $\mathrm{R}_{j}$ and the other relay. Each relay uses all the $M_{r}$ antennas to receive messages but only chose the best antenna to transmit signals for simplicity. The index of such a transmit antenna is defined as $r_{j}$ at $\mathrm{R}_{j}$. Note that this antenna selection method can preserve the diversity gain achieved by all the antennas at each relay in the high SNR regions.

The proposed protocol consists of two stages, initialization and data transmission.

\subsection{Initialization}

During the initialization stage, each relay channel is assumed to have the knowledge of its incoming and outgoing channel state information (CSI). Based on the sourcerelay CSI, using the relay selection method in [8], we known that $\mathrm{R}_{j}$ can decode the messages from $S_{i}$ when the source-relay channel satisfies $\log \left(1+\rho\left|\mathbf{h}_{i, R j}\right|^{2}\right)>\tilde{R}$. Here, $\log (\cdot)$ is taken to base 2, $\rho$ denotes the transmit signal-tonoise ratio (SNR), where we have assumed that all transmitters are under the same and fixed power constraint, $|\cdot|$ denotes the Euclidean norm of a vector, and $\tilde{R}$ denotes the number of bits in each codeword transmitted by sources ${ }^{\mathrm{a}}$. If $\mathrm{R}_{j}$ can correctly decode the messages from both sources, i.e., $\left|\mathbf{h}_{i, R j}\right|^{2} \geq \frac{2^{\tilde{R}}-1}{\rho}$ for $\forall i \in\{1,2\}$, it will broadcast a onebit indicator, which is denoted as ACK1. Otherwise, $\mathrm{R}_{j}$ broadcasts a one-bit indicator NACK1, assumed that all the other nodes can correctly receive each feedback signal. Denote $A_{k}$ as the situation in which $k$ relays broadcast ACK1 signals. On the other hand, based on the relaydestination CSI, each relay can determine the index of the best antenna as $r_{j} \triangleq \arg \max _{r=1 \ldots M_{r}}\left|g_{j, r}\right|^{2}$.

For situation $A_{2}$ in which both relays broadcast ACK1 signals, two sub-events will be further considered. According to the source-relay/inter-relay $\mathrm{CSI}_{\mathrm{N}} \mathrm{R}_{j}$ will determine whether it can use ZF detection to separate the mixture of two unknown streams which are from $S_{j}$ and the other relay, respectively. For the $\mathrm{ZF}$ receiver, the qualification criterion [26] is $\left[\left(\mathbf{H}_{R j}^{H} \mathbf{H}_{R j}\right)^{-1}\right]_{k, k}<\rho / 2^{\tilde{R}-1}$, where $[\mathbf{X}]_{k, k}$ represents the $k$-th diagonal element of matrix $\mathbf{X}, \mathbf{H}_{R j}=$ $\left[\begin{array}{ll}\beta_{1} \mathbf{h}_{2, r_{2}} & \alpha_{1} \mathbf{h}_{1, R 1}\end{array}\right]$ for $j=1$, and $\left[\beta_{1} \mathbf{h}_{1, r_{1}} \alpha_{1} \mathbf{h}_{2, R 2}\right]$ for $j=2$. Note that $\alpha_{i}$ and $\beta_{i}$ are the power weighting factors at the sources and relays, respectively, which are constrained by $0<\alpha_{i}, \beta_{i}<1$ and $\sum_{i=1}^{2} \alpha_{i}^{2}=\sum_{i=1}^{2} \beta_{i}^{2}=1$. If $\mathrm{R}_{j}$ satisfies this ZF condition, it will send back another one-bit 


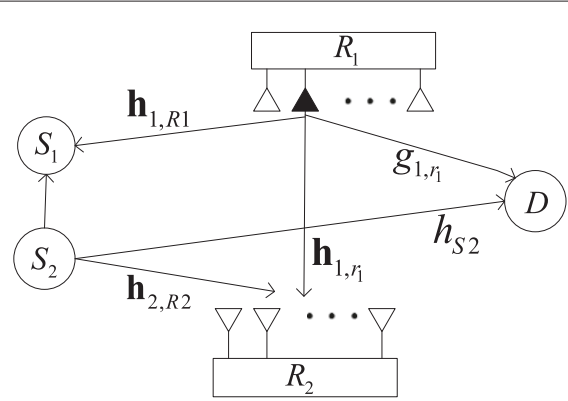

(a) When $S_{2}$ and $R_{1}$ transmit signals

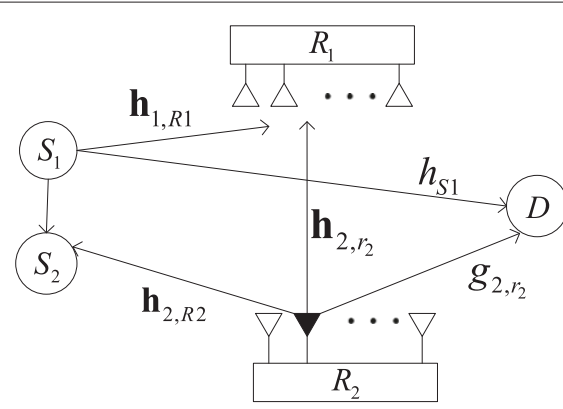

(b) When $S_{1}$ and $R_{2}$ transmit signals

Figure 1 The system model and ARDF process at two successive time slots (a-b). Each relay is equipped with multiple antennas. The antenna marked with black color for each relay is selected as the transmit antenna.

indicator ACK2. Otherwise, it sends back NACK2. Let $E$ denote the event that both relays send back ACK2 signals, and such an event can be represented as [26]

$$
E \triangleq\left\{\log \left(1+\frac{\rho}{\left[\left(\mathbf{H}_{R j}^{H} \mathbf{H}_{R j}\right)^{-1}\right]_{k, k}}\right)>\tilde{R}, \forall k, j \in\{1,2\}\right\},
$$

Otherwise, the system lies in event $\bar{E}$ if any relay sends back a NACK2 signal.

\subsection{Cooperative transmission}

Based on the initialization stage, the transmission is divided into four different modes according to the indicators sent back from the two relays. The flow chart of the proposed protocol is shown in Figure 3, where each of the sources is assumed to correctly decode the messages of the other source, which is valid for high SNR regions as shown in [5]. In the next paragraphs, we will describe the cooperative transmission mode for each situation in details.

For situation $A_{0}$ in which both relays send back NACK1 signals, we can only utilize the mutual cooperation between the two sources. In one cooperative data frame, each source intends to send $L$ codewords, and the system works in mode 1 . In time slot $n(2 \leq n \leq 2 L)$, $\mathrm{S}_{i}$ will transmit a mixed message ${ }^{\mathrm{b}} \alpha_{2} x(n-1)+\alpha_{1} x(n)$ and the other source listens, where $i=2$ if $n$ is even and $i=1$ if $n$ is odd. The data rate per channel use here is $\frac{2 L}{2 L+1} \tilde{R}$.

For situation $A_{1}$ in which only one relay sends back ACK1 signal, we assume that $R_{1}$ is this relay without loss of generality. Each source intends to transmit only one codeword, and the system works in mode 2. For the first two time slots, the transmission process is the same as that in mode 1 , and $\mathrm{R}_{1}$ can correctly decode $x(1)$ and $x(2)$ since it sends back ACK1. In the third time slot, $\mathrm{S}_{1}$ and $\mathrm{R}_{1}$ will retransmit $x(2)$ and $x(1)$, respectively. In the fourth time slot, $\mathrm{R}_{1}$ will retransmit $x(2)$. The transmission data rate in this situation is $\frac{2}{4} \tilde{R}$.

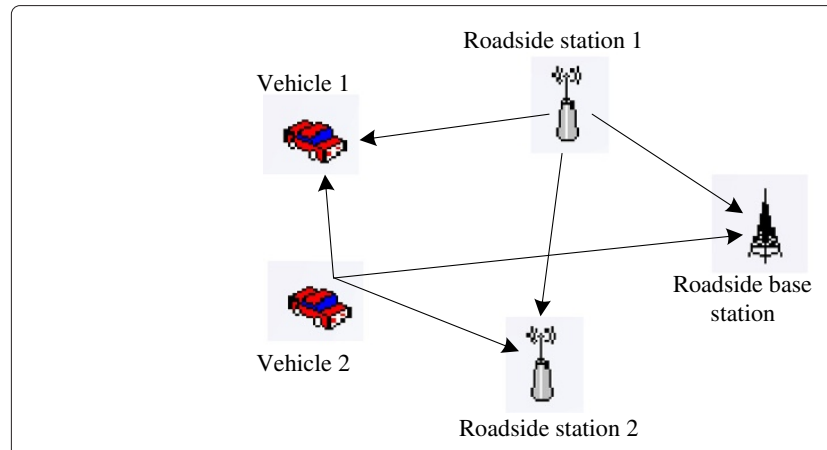

(a) When vehicle 2 and roadside station 1 transmit signals

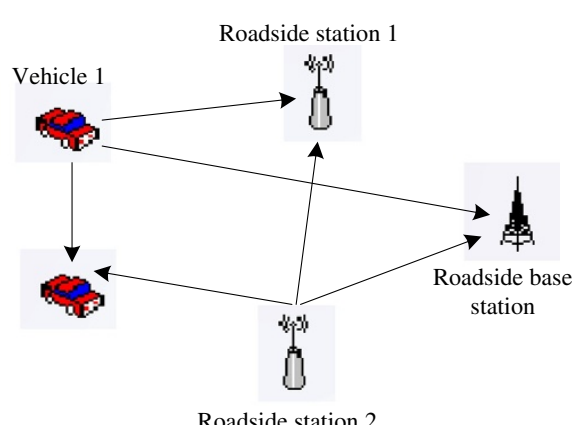

(b) When vehicle 1 and roadside station 2 transmit signals

Figure 2 The application of the system model in Figure 1 to the wireless vehicular networks (a-b). Two vehicles communicate with a common roadside base station with the help two roadside stations. 


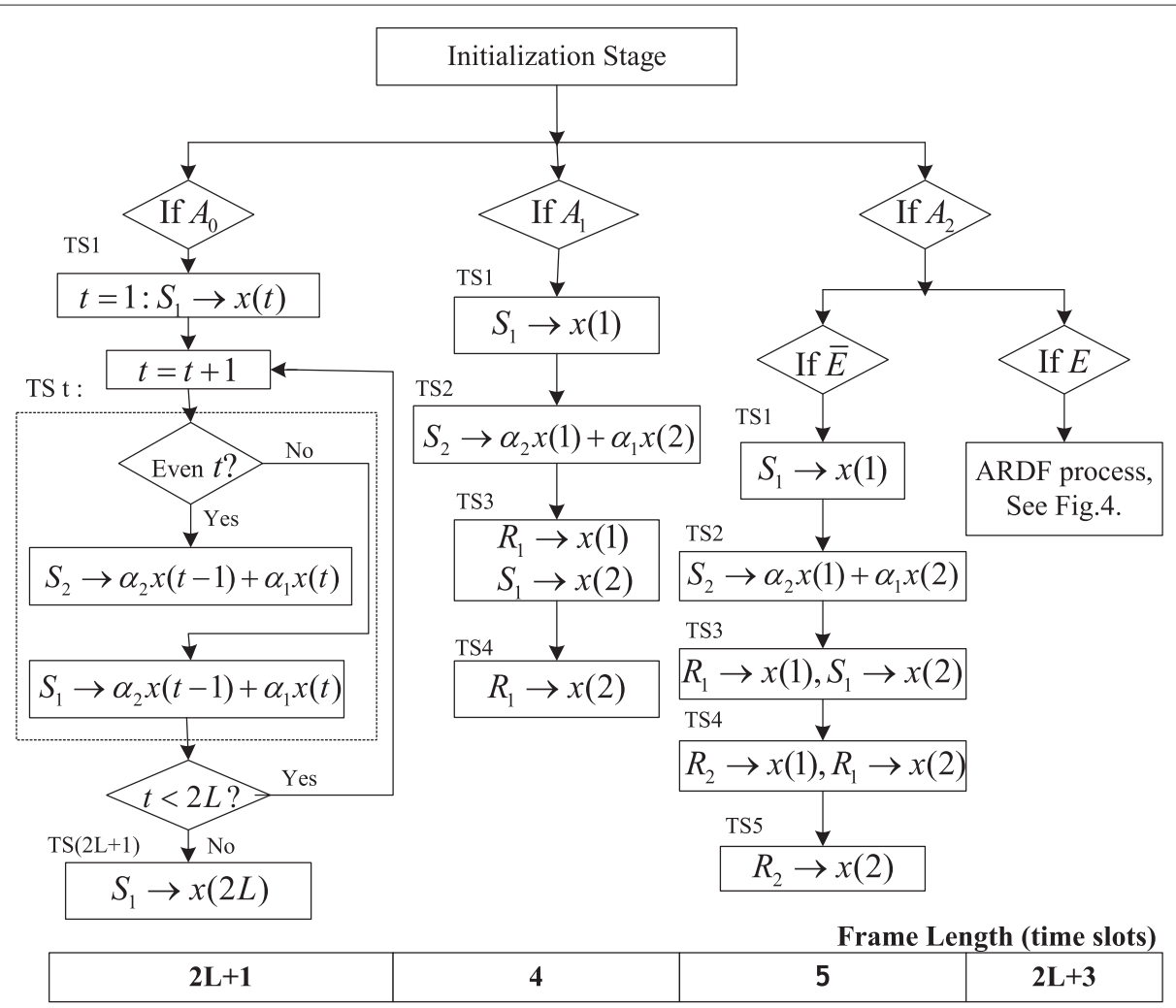

Figure 3 The flow chart for the transmission stage. Note that $A_{k}$ denotes the situation that $k$ relays broadcast ACK 1 signals, $k=0,1,2$. The event $E$ denotes that both relays broadcast ACK2 signals, otherwise the system lies in Event $\bar{E}$. $L$ is used to denote the number of codewords transmitted by each source in the corresponding data frame. $\alpha_{i}$ is power allocation factor at the sources, $i=1,2$.

For situation $A_{2}$, if the event $\bar{E}$ occurs, the system works in mode 3 . The transmission scheduling during the first three time slots is the same as that in mode 2. Then, during the fourth time slot, $\mathrm{R}_{1}$ and $\mathrm{R}_{2}$ will retransmit $x(2)$ and $x(1)$, respectively. During the fifth time slot, $\mathrm{R}_{2}$ retransmits $x(2)$. The data rate for the event $\bar{E}$ is $\frac{2}{5} \tilde{R}$. For the event $E$, both relays broadcast ACK2 signals. As shown in Equation (2), each relay can correctly decode two unknown codewords transmitted by a source and the other relay, so the ARDF transmission process can be performed. The two sources will transmit $2 L$ codewords to the destination during $2 L+3$ time slots, so the data rate is $\frac{2 L}{2 L+3} \tilde{R}$. The details of such an ARDF process are put into in to the next section, i.e., Section 3 , and the system works in mode 4.

From the protocol description, the transmission mode and equivalent channel matrix for each situation can be easily shown in Table 1.

\section{Alternative-relaying DF process}

\subsection{Transmission process}

The proposed ARDF transmission process follows the concept of successive relaying [13], where the two half- duplex relays alternatively retransmit the source messages to mimic a full-duplex node. As shown in Figure 1, the sending source sends a superposition codeword $\alpha_{2} x$ $(n-3)+\alpha_{1} x(n)$ in any one time slot $n(n>3)$, where $x(n)$ is its own codeword and $x(n-3)$ is the codeword it received from the other source in time slot $n-3$. At the same time, the sending relay will transmit a superposition codeword $\beta_{2} x(n-1)+\beta_{1} x(n)$, where $x(n-1)$ and $x(n-2)$ are codewords it received from the source during the previous two time slots. The other source (i.e., the listening source) and the other relay (i.e., the listening relay) listen to the transmissions in this time slot. In the next time slot, i.e., time slot $n+1$, both the two sources and relays will exchange their working roles, where the listening ones become the sending ones and the sending ones become the listening ones. At the sources, different to [5,6], the sending source retransmit the codeword $x(n-3)$ instead of $x(n-1)$ in time slot $n$. This is because we have arranged the sending relay to transmit $x(n-1)$, so such a codeword should not be transmitted by the sending source again in this time slot to avoid the potential mutual cancellation. In the proposed ARDF process, each codeword can be transmitted by both the sources and relays during four successive time slots, which ensures that the 'full' cooperation can be achieved. 
Table 1 The transmission modes for each situation, where $y_{i}, n_{i} \in \mathcal{C}^{i}, i=4,5,2 L+1,2 L+3 ; x_{j} \in \mathcal{C}^{j}, j=2,2 L$

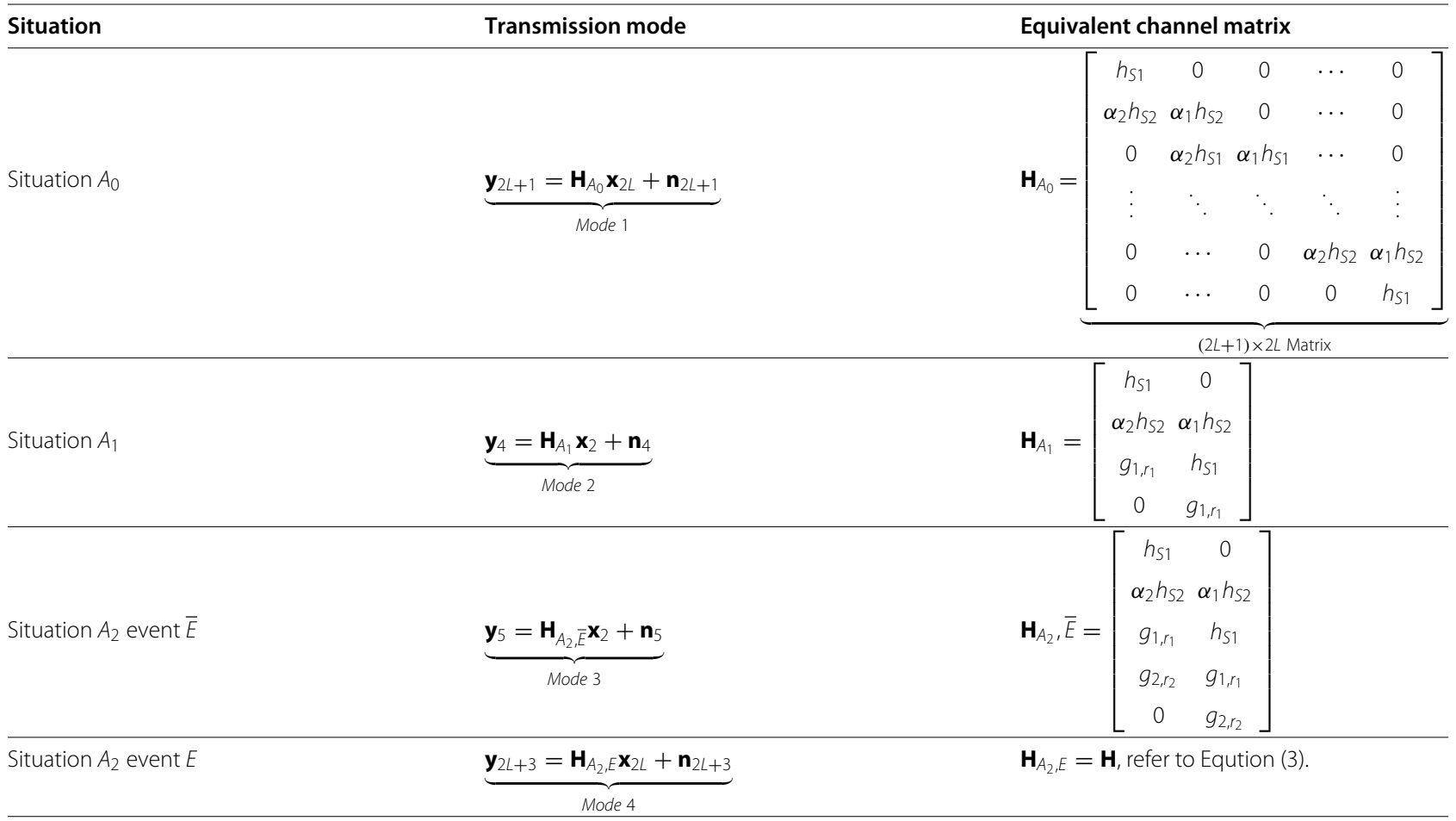

In the following paragraphs, we will describe the transmission process during the four successive time slots in details.

Assume $n$ to be odd without loss of generality. In time slot $n(1 \leq n \leq 2 L)$, the sending source $S_{1}$ first transmits $x(n)$, while $\mathrm{R}_{1}$ and $\mathrm{S}_{2}$ decode $x(n)$ from $\mathrm{S}_{1}$. In time slot $n+1, \mathrm{R}_{1}$ is the sending relay and uses its best antenna to retransmit $x(n) ; \mathrm{R}_{2}$ receives $x(n)$ from $\mathrm{R}_{1}$. In time slot $n+2$, $\mathrm{R}_{2}$ becomes the sending relay and uses its best antenna to repeat $x(n)$. In time slot $n+3$, the sending source $S_{2}$ retransmits $x(n)$ again. Therefore, during such four successive time slots, $x(n)$ can be transmitted four times, by both two sources and relays. Similar to the transmission of $x(n)$, the codeword of $S_{2}, x(n+1)$, will be transmitted by $S_{2}, R_{2}, R_{1}$, and $S_{1}$ in time slot $n+1, n+2, n+3$, and $n+4$, respectively. When a transmitting node wishes to transmit two codewords simultaneously, it will transmit the superposition of these two codewords and use an amplitude factor to constrain the power of each codeword. Figure 4 illustrates the whole ARDF transmission process, where $2 L+3$ time slots are required for a cooperative data frame.

From the above ARDF transmission process, one can observe that the listening relay may need to decode at most two unknown codewords in a time slot. For instance, during time slot $n(2 \leq n \leq 2 L)$, in addition to the new source codeword $x(n)$, the listening relay also does not know $x(n-1)$ transmitted by the sending relay. This is because it is transmitting during the previous time slot and cannot simultaneously receive the source codeword $x(n-1)$ due to the half-duplex constraint. After canceling the known codewords, this relay will decode two independent unknown codewords, i.e., $x(n)$ and $x(n-1)$, from one source and the other relay, respectively. Such a transmission mode mimics a two-user MAC with two single-antenna transmitters and one $M_{r}$ antenna receiver. At each relay, simple linear zero-forcing detection approaches can be used to separate the source message and the interference from the other relay, where each relay is equipped with multiple antennas and therefore has the capability to separate the messages under the ZF condition in Equation (2).

\subsection{Data model}

Note that we have used a fixed power allocation strategy in each time slot, and the use of more advanced adaptive power allocation shall yield better outage performance at finite SNRs. However, for the infinite SNR region, the precise power allocation does not affect the final DMT result [27].

For the ARDF process with respect to situation $A_{2}$ event $E$, the data model can be expressed as $\mathbf{y}=\mathbf{H x}+\mathbf{n}$, where $\mathbf{x}=[x(1), \cdots, x(2 L)]^{T}$, which is the codeword vector of the two sources transmitted during a data frame; $\mathbf{y}=[y(1), \cdots, y(2 L+3)]^{T}$, which is the observation vector at the destination during a data frame that lasts $2 L+3$ time 

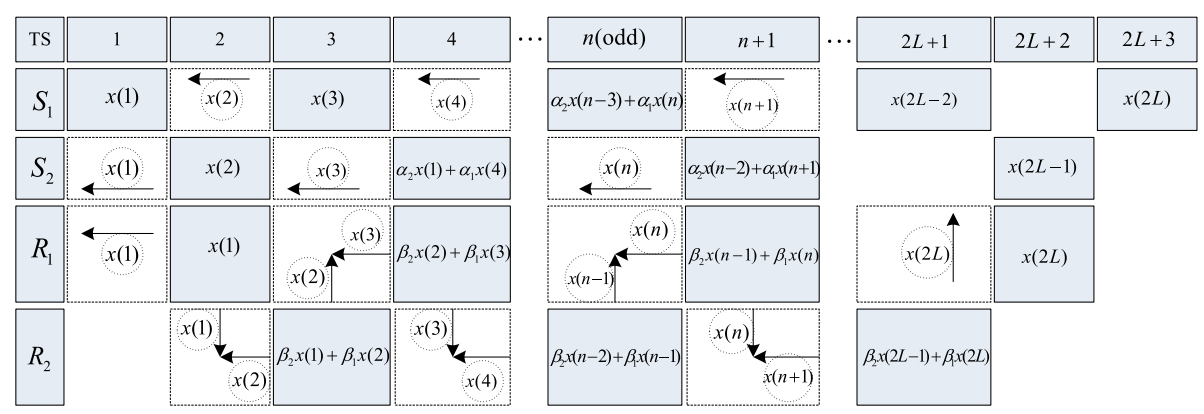

Figure 4 The ARDF process during each time slot (TS). Here $n$ is an odd number and $5 \leq n \leq 2 L-1, L \geq 3$. The solid blocks denote that the sending relays transmit the codewords using the best antenna. Dashed blocks denote that the listening relays receive signals using all the antennas, and each codeword within the dashed circle is an unknown codeword to be decoded. The horizontal and vertical arrows imply that a node receives the corresponding codewords from sending source and the sending relay, respectively.

slots; $\mathbf{n}=[n(1), \cdots, n(2 L+3)]^{T}$, which is the Gaussian additive noise vector; and the $(2 L+3) \times(2 L)$ equivalent channel matrix is as follows:

$\mathbf{H}=\left[\begin{array}{ccccccc}h_{S 1} & 0 & 0 & 0 & 0 & \ldots & 0 \\ g_{1, r_{1}} & h_{S 2} & 0 & 0 & 0 & \ldots & 0 \\ \beta_{2} g_{2, r_{2}} & \beta_{1} g_{2, r_{2}} & h_{S 1} & 0 & 0 & \ldots & 0 \\ \alpha_{2} h_{S 2} & \beta_{2} g_{1, r_{1}} & \beta_{1} g_{1, r_{1}} & \alpha_{1} h_{S 2} & 0 & \ldots & 0 \\ 0 & \alpha_{2} h_{S 1} & \beta_{2} g_{2, r_{2}} & \beta_{1} g_{2, r_{2}} & \alpha_{1} h_{S 1} & \ldots & 0 \\ \vdots & \ddots & \ddots & \ddots & \ddots & \ddots & \vdots \\ 0 & \ldots & 0 & \alpha_{2} h_{S 2} & \beta_{2} g_{1, r_{1}} & \beta_{1} g_{1, r_{1}} & \alpha_{1} h_{S 2} \\ 0 & \ldots & 0 & 0 & h_{S 1} & \beta_{2} g_{2, r_{2}} & \beta_{1} g_{2, r_{2}} \\ 0 & \ldots & 0 & 0 & 0 & h_{S 2} & g_{1, r_{1}} \\ 0 & \ldots & 0 & 0 & 0 & 0 & h_{S 1}\end{array}\right]$.

\section{Diversity-multiplexing trade-off and numerical results}

In this section, the analytical performance evaluation of the proposed protocol will be first shown by using DMT. Then, the Monte Carlo simulations will be carried out to demonstrate the outage performance of the proposed cooperative ARDF protocol.

\subsection{Achievable diversity-multiplexing trade-off}

Firstly, recall that the diversity and multiplexing gain are defined as [7]

$$
d \triangleq-\lim _{\rho \rightarrow \infty} \frac{\log \left[P_{e}(\rho)\right]}{\log \rho} \quad \text { and } \quad r \triangleq \lim _{\rho \rightarrow \infty} \frac{R(\rho)}{\log \rho},
$$

where $P_{e}$ is the ML detection error probability and $R$ is the target data rate per channel use. Following similar steps in [22], it can be proved that the ML error probability in the MAC scenario can be tightly bounded by the outage probability at high SNR. So the outage probability will be focused in this paper. When $\lim _{\rho \rightarrow \infty} \frac{\log f(\rho)}{\log \rho}=d, f(\rho)$ is said to be exponentially equal to $\rho^{d}$, denoted as $f(\rho) \doteq \rho^{d}(\dot{\leq}$

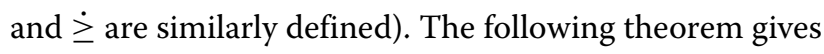
the final DMT performance of the proposed cooperative protocol.

Theorem 1. When each relay is equipped with at least two antennas, i.e., $M_{r} \geq 2$, the achievable DMT of the proposed ARDF protocol for the two-user and two-relay CMA scenario can be expressed as

$$
d^{*}(r)=\left(2 M_{r}+2\right)\left(1-\frac{2 L+3}{2 L} r\right)^{+},
$$

where $(x)^{+}$denotes $\max \{x, 0\}$.

Proof 1. We have briefly provided the proof of this theorem in [28] without formal derivations. In this journal paper, the details of the proof for this theorem will be blue presented in Appendix 2.

- Remark 1: The optimal MISO DMT for the addressed CMA scenario can be expressed as

$$
d_{M I S O}(r)=\left(2 M_{r}+2\right)(1-r), \quad 0 \leq r \leq 1 .
$$

According to Equation (5), when the frame length $L$ is sufficiently large, the achievable DMT of the proposed scheme yields $\frac{2 L+3}{2 L} \approx 1$, which demonstrates that the optimal MISO trade-off $d_{\text {MISO }}(r)$ can be asymptotically approached by the proposed scheme.

- Remark 2: The achievable DMT of the CMA schemes in $[4,5]$ can be written as

$$
d_{C M A}(r)=M(1-r), \quad 0 \leq r \leq 1,
$$

where $M$ denotes the number of the sources. When there only exist two active users in a network, $d_{C M A}(r)=2(1-r)$. Hence, the schemes in $[4,5]$ cannot perform well for a small number of sources, whereas the proposed ARDF-CMA scheme can 
further enhance the reception robustness by exploiting the relay nodes.

- Remark 3: On the other hand, the R-CMA protocol in [18] can be straightforwardly extended to the two-user two-relay scenario considered in this paper. It means that each relay apply a simple antenna selection, and the DMT result presented in Equation (7) in [18] can be accordingly updated as

$$
d_{R_{-} C M A}(r)=(1-r)+M_{r}(2-4 r)^{+}, \quad 0 \leq r \leq 1 .
$$

Compared to the R-CMA scheme, the proposed scheme achieves one more diversity gain, because it exploits the help of two $M_{r}$-antenna relays while preserves the cooperation between two sources. Although the proposed protocol can only achieve a maximal multiplexing gain $\frac{2 L}{2 L+3}$ while the schemes in [18] can achieve 1 , the difference is negligible for a large $L$.

Figure 5 illustrates the achievable DMTs of various cooperative schemes. As can be seen from this figure, the proposed scheme can approach the optimal MISO upper bound for a sufficiently large $L$, and hence, outperforms the comparable ones in most multiplexing range.

\subsection{Numerical results}

In this section, some numerical results based on the Monte Carlo simulations are provided for the addressed CMA scenario. The targeted data rate is set as 2 or 4 bits per channel use (BPCU). Each relay is equipped with two

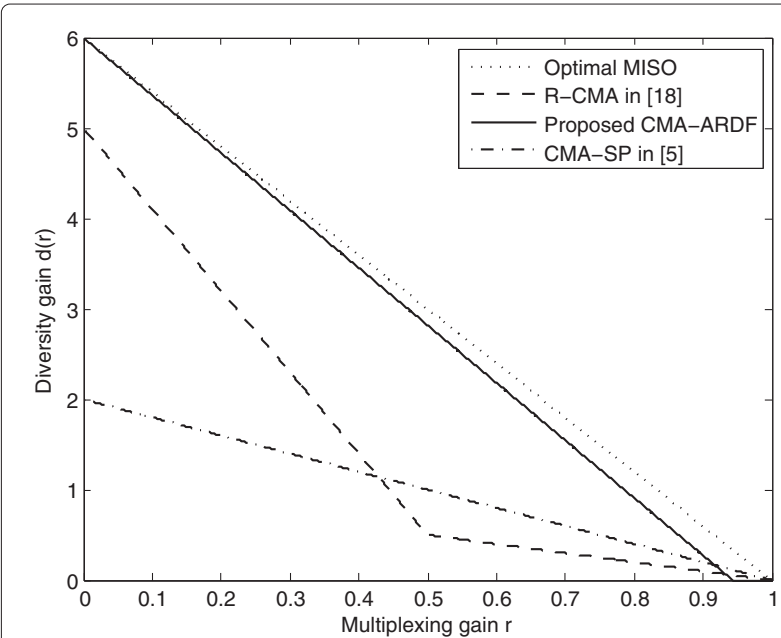

Figure 5 The DMT performance of different protocols for addressed CMA channel. Here each relay is equipped with two antennas. For the proposed protocol, the number of codewords transmitted by each source in one cooperative data frame is fixed as $L=25$. The two sources communicate with the destination using TDMA for all the protocols. antennas, i.e., $M_{r}=2$. For the proposed CMA-ARDF protocol, set $L=25, \alpha_{1}=\beta_{1}=0.87$ for simplicity. Note that all the channels are assumed to be Rayleigh fading with unit variance and all the nodes have the same transmit power.

In addition to the superposition-coding-based CMA in [5], the R-CMA scheme in [18] is also used to compare with the proposed ARDF protocol. As shown in Figure 6, ARDF has limited performance at low SNR. This is because the performance of the proposed scheme is much more sensitive to the source-relay/inter-relay channel condition, and the outage event is prone to occur at each relay at low SNR, which results in some degradation to the outage performance. However, the proposed scheme achieves the smallest outage probability among the comparative ones when we increase SNR. This performance gain is due to the fact that the proposed ARDF scheme can exploit the available relays to provide an extra dimension to improve the outage performance, whereas the scheme in [5] only considers the cooperation between the two sources and the R-CMA scheme in [18] does not consider the mutual source cooperation. Therefore, larger diversity gains can be achieved by the proposed transmission protocol, which guarantee the superior performance particularly at high SNR. In Figure 7, different antenna numbers, i.e., $M_{r}$, is consider at each relay to demonstrate the outage performance of the proposed scheme. One can observe that increasing the number of relay antennas can further improve the robustness of the transmission scheme, since more diversity gains can be provided.

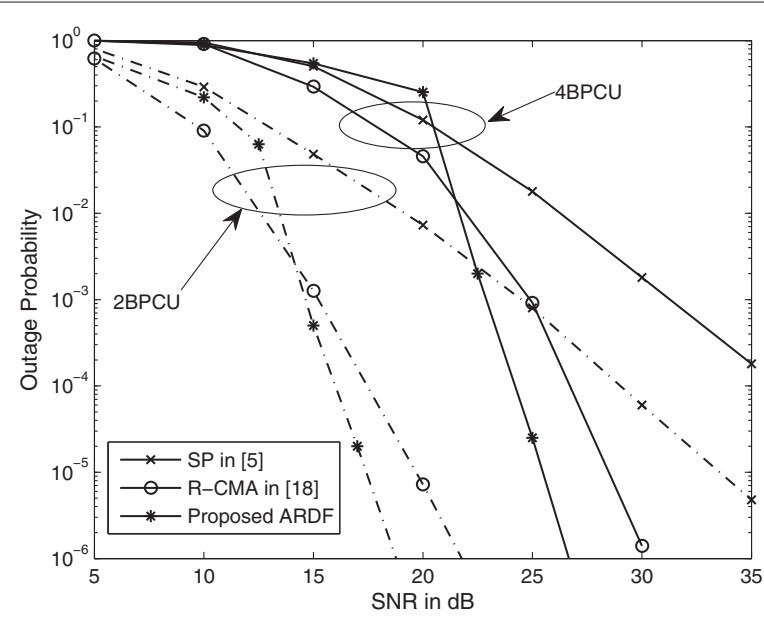

Figure 6 The outage probability for the proposed protocol, the superposition cooperative scheme in [5] and non-cooperative transmission. Note that each relay is equipped with two antennas. For the proposed protocol, we set $L=25$. The power allocation at the sources for both the proposed ARDF scheme and the SP scheme in [5] is fixed as $\alpha_{1}^{2}=0.87$. Moreover, we set $\beta_{1}^{2}=0.87$ at the relays. 


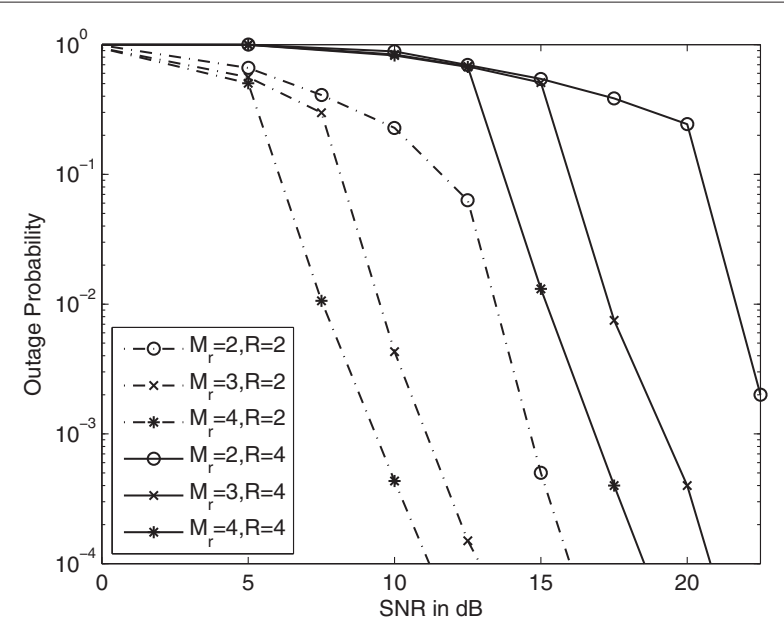

Figure 7 The outage probability for the proposed ARDF scheme versus antenna number $M_{r}$ at relays. Set $L=25, \alpha_{1}^{2}=\beta_{1}^{2}=0.87$.

\section{Conclusions}

In this paper, a new cooperative transmission protocol has been proposed for a CMA scenario in the wireless vehicular networks. Without the use of assumptions of strong inter-relay channel in [13], the relay reuse has still been realized using the linear ZF detection at the halfduplex relays to combat a general inter-relay interference. In addition, to fully exploit the cooperation between all the transmitters, the superposition coding strategy has also been carefully applied at both sources and relays . To evaluate the spectral efficiency, an achievable DMT of the proposed scheme was developed, which demonstrated that the proposed ARDF protocol can outperform the existing related schemes and approximately achieve the optimal MISO upper bound. The derived analytical result and the numerical results have demonstrated that the proposed scheme has a better performance in comparison with the existing related schemes in most conditions. The impact of adaptive power allocation has not been analyzed due to the high SNR considered in this paper, where a promising future direction is to carry out the study for the optimal design of power allocation to improve the overall system throughput at intermediate SNR.

\section{Endnotes}

${ }^{a}$ For a particular situation, the data rate should be $\frac{\tilde{R} \times N_{x}}{N_{t}}$, where $N_{x}$ and $N_{t}$ are the number of transmitted codewords and the number of required time slots, respectively. So the targeted data rate $R$ (i.e., the average data rate) can be calculated from $\tilde{R}$. We will provide more discussions about the relationship between the two rates later in this paper.

${ }^{\mathrm{b}}$ In this paper, $x(n)$ denotes the new source codeword sent by $S_{i}(i=1,2)$ in time slot $n(1 \leq n \leq 2 L)$. Obviously, the mapping criterion is $x(n)=\left\{\begin{array}{cl}s_{1}\left(\frac{n+1}{2}\right), & \text { if } n \text { is odd } \\ s_{2}\left(\frac{n}{2}\right), & \text { if } n \text { is even }\end{array}\right.$, where $s_{i}(l)$ denotes the $l$-th $(1 \leq l \leq L)$ codeword of $S_{i}$ in one cooperative frame.

\section{Appendices \\ Appendix 1 \\ Preliminary results}

We first present four lemmas and their proof steps in this section. These results will be useful for the proofs of Theorem 1 in the next section.

Lemma 2. By assuming quasi-static and flat Rayleighfading channels, the probability of each situation $A_{k}$ with $k$-qualified relays broadcasting ACK1 signals can be expressed as $P\left(A_{k}\right) \doteq \rho^{-(2-k) M_{r}(1-\tilde{r})}$, where $\tilde{r}=$ $\lim _{\rho \rightarrow \infty} \frac{\tilde{R}(\rho)}{\log \rho}$.

Lemma 3. By assuming quasi-static and flat Rayleighfading channels and $\alpha_{1}=\beta_{1}=\sqrt{\frac{1}{2}}$ for simplicity, the probability of the event $E$ that both relays can successfully perform ZF detection and broadcast ACK2 signals, as expressed in Equation (2), can be bounded as

$$
1-\frac{4 \gamma^{M_{r}-1}}{\left(M_{r}-1\right) !} \leq P(E) \leq 1-\frac{2 \gamma^{M_{r}-1}}{\left(M_{r}-1\right) !},
$$

where $\gamma=\frac{2^{\tilde{R}}-1}{\rho \alpha_{1}^{2} \delta^{2}}, \delta^{2}$ is the variance of each channel coefficient. Furthermore, the probability of the event $\bar{E}$ can be obviously obtained as $P(\bar{E}) \doteq \rho^{-\left(M_{r}-1\right)(1-\tilde{r})}$.

Lemma 4. When $X_{1}$ and $X_{2}$ are subjected to exponential distribution, the probability can be approximated as $P\left(X_{1} X_{2}<a\right) \approx-a \ln a$, where $a>0$ and is sufficiently small.

Lemma 5. Assuming that $z=\max \left\{z_{1}, \cdots, z_{M_{r}}\right\}$, where $z_{i}$ is exponentially distributed with unit variance, the expectation of $e^{-c z}(c>0)$ can be revealed as $\epsilon\left[e^{-c z}\right]=$ $\frac{M_{r} !}{\Pi_{i=1}^{M}(c+i)}, \epsilon[\cdot]$ denotes the expectation of a random variable.

The proofs steps of the above lemmas are provided in the following section.

\section{Proof of Lemma 2}

Denote $B$ as the event that a relay $\mathrm{R}_{j}$ is not qualified, i.e., it can be expressed as the event: $B \triangleq$ $\bigcup_{i=1}^{2}\left\{\log \left(1+\rho \alpha_{1}^{2}\left|\mathbf{h}_{i, R j}\right|^{2}\right)<\tilde{R}\right\}$. The probability of the event $B$ can be easily calculated as $P(B) \doteq \rho^{-M_{r}(1-\tilde{r})}$. 
Thus, the probability of each situation $A_{k}$ can be expressed as follows:

$$
P\left(A_{k}\right) \doteq\left(\begin{array}{l}
2 \\
k
\end{array}\right)[1-P(B)]^{k}[P(B)]^{2-k} \doteq \rho^{-(2-k) M_{r}(1-\tilde{r})}
$$

\section{Proof of Lemma 3}

Consider a signal model in which the $r_{2}$-th antenna of $\mathrm{R}_{2}$ transmits a message $\tilde{s}_{1}$ with a power lever $\beta_{1}^{2}$ while the first source $S_{1}$ broadcasts a message $\tilde{s}_{2}$ with a power lever $\alpha_{1}^{2}$. We set $\alpha_{1}=\beta_{1}=\sqrt{\frac{1}{2}}$ for ease of explanation. Such a signal model can be found in most time slots with odd numbers in situation $A_{2}$ event $E$. At this time, the signal vector received by $R_{1}$ can be written as $\mathbf{r}_{R 1}=\mathbf{H}_{R 1} \tilde{\mathbf{s}}+\mathbf{w}_{1}$, where $\mathbf{r}_{R 1}, \mathbf{w}_{1} \in \mathcal{C}^{M_{r}}$, $\mathbf{H}_{R 1}=\left[\begin{array}{ll}\beta_{1} \mathbf{h}_{2, r_{2}} & \alpha_{1} \mathbf{h}_{R 1}\end{array}\right], \tilde{\mathbf{s}}=\left[\tilde{s}_{1}, \tilde{s}_{2}\right]^{T}$, and $\mathbf{w}_{1}$ is the noise vector. This transmission model can be recognized as a special MIMO system with two transmit, and $M_{r}$ receive antennas when ZF detection is applied. Denote the event as $E_{R 1} \triangleq\left\{\log \left(1+\frac{\rho}{\left[\left(\mathbf{H}_{R 1}^{H} \mathbf{H}_{R 1}\right)^{-1}\right]_{k, k}}\right)>\tilde{R}, \forall k \in\{1,2\}\right\}$. Applying ZF detection at the receiver and according to [26], the probability of $E_{R 1}$ can be expressed as

$$
P\left(E_{R 1}\right)=1-\frac{2 \gamma^{M_{r}-1}}{\left(M_{r}-1\right) !}
$$

when $\rho$ is sufficiently large, where $\gamma=\frac{2^{\tilde{R}}-1}{\rho \alpha_{1}^{2} \delta^{2}}$. Note that the similar ZF decoding can be applied at $R_{2}$ when $R_{1}$ and $S_{2}$ are transmitting messages at the same time, and $E_{R 2}$ is similarly defined as $E_{R 2} \triangleq$ $\left\{\log \left(1+\frac{\rho}{\left[\left(\mathbf{H}_{R 2}^{H} \mathbf{H}_{R 2}\right)^{-1}\right]_{k, k}}\right)>\tilde{R}, \forall k \in\{1,2\}\right\}$, whose probability is the same as $P\left(E_{R 1}\right)$, i.e.,

$$
P\left(E_{R 2}\right)=1-\frac{2 \gamma^{M_{r}-1}}{\left(M_{r}-1\right) !} .
$$

Then, the probability of the event $E$ defined in Equation (2) can be revealed as $P(E)=P\left(E_{R 1} \bigcap E_{R 2}\right)$, where the events $E_{R 1}$ and $E_{R 2}$ are not strictly independent. Since reciprocal channel is assumed, the channel vectors $\mathbf{h}_{1, r_{1}}$ and $\mathbf{h}_{2, r_{2}}$ have one common element which is the channel coefficient between the $r_{1}$-th antenna of $\mathrm{R}_{1}$ and the $r_{2}$-th antenna of $\mathrm{R}_{2}$, so that it is difficult to obtain the accurate value of $P(E)$. However, when we notice that $P(E)$ can be bounded as $P\left(E_{R 1}\right)+P\left(E_{R 2}\right)-1 \leq P(E) \leq P\left(E_{R 1}\right)$, we have

$$
P\left(E_{R 1}\right)-\frac{2 \gamma^{M_{r}-1}}{\left(M_{r}-1\right) !} \leq P(E) \leq P\left(E_{R 1}\right) .
$$

According to Equation (A.3), Lemma 3 can be proved.

\section{Proof of Lemma 4}

Assume $X_{1}$ and $X_{2}$ to be independently exponentially distributed with unit variance without loss of generality. Let $D=\left\{\left(x_{1}, x_{2}\right) \in \mathbb{R}^{2+} \mid x_{1} x_{2}<a\right\}$ and $f_{x_{1} x_{2}}\left(x_{1}, x_{2}\right)=$ $f_{x_{1}}\left(x_{1}\right) f_{x_{2}}\left(x_{2}\right)$ which is the joint density of $\left\{x_{1}, x_{2}\right\}$, where $f_{x_{i}}\left(x_{i}\right)$ denotes $X_{i}$ 's probability density function (PDF) $f_{x_{i}}=$ $e^{-x_{i}}, i=1,2$. Then, the probability $P\left(X_{1} X_{2}<a\right)$ can be expressed as

$$
\begin{aligned}
\Phi_{a} & =P\left(X_{1} X_{2}<a\right)=\iint_{D} f_{x_{1} x_{2}}\left(x_{1}, x_{2}\right) \mathrm{d} x_{1} \mathrm{~d} x_{2} \\
& =\int_{0}^{+\infty} e^{-x}\left(1-e^{-a / x}\right) \mathrm{d} x \\
& =\sum_{i=1}^{\infty} \frac{(-1)^{i+1}}{i !} a^{i} \int_{0}^{+\infty} \frac{e^{-x}}{x^{i}} \mathrm{~d} x .
\end{aligned}
$$

Now, the function $\Phi_{a}(u)$ is first defined as

$$
\Phi_{a}(u)=\sum_{i=1}^{\infty} \frac{(-1)^{i+1}}{i !} a^{i} B_{i}(u)
$$

where $B_{i}(u)=\int_{u}^{+\infty} \frac{e^{-x}}{x^{i}} \mathrm{~d} x$. Then, the improper integral $\Phi_{a}$ can be calculated as $\Phi_{a}=\lim _{u \rightarrow 0^{+}} \Phi_{a}(u)$.

Moreover, from [29] (Equation 3.351.4), $B_{i}(u)$ can be calculated by the exponential integral function as

$$
\begin{aligned}
B_{i}(u)= & (-1)^{i} \frac{\operatorname{Ei}(-u)}{(i-1) !} \\
& +e^{-u} \sum_{n=0}^{i-2} \frac{(-1)^{n} u^{-(i-1-n)}}{(i-1)(i-2) \cdots(i-1-n)},
\end{aligned}
$$

where $i \geq 2$, and exponential integral function can be shown as $\operatorname{Ei}(-u)=\ln (u)+\sum_{k=1}^{\infty} \frac{(-u)^{k}}{k \cdot k !}$. Now that, $B_{i}(u)$ can be obtained as

$$
\frac{B_{i}(u)}{e^{-u}}=\left\{\begin{array}{lll}
-\ln (u)+o(1) & \text { if } & i=1 \\
\frac{(-1)^{i} \ln (u)}{(i-1) !}+\sum_{n=0}^{i-2} \frac{(-1)^{n} u^{-(i-1-n)}}{(i-1)(i-2) \cdots(i-1-n)}+o(1) & \text { if } & i \geq 2
\end{array},\right.
$$

where $o(1) \rightarrow 0$ when $u \rightarrow 0^{+}$. By substituting $B_{i}(u)$ into Equation (A.7) and rearranging the infinite series, $\Phi_{a}(u)$ can be rewritten as

$$
\Phi_{a}(u)=e^{-u} \sum_{n=0}^{\infty} \phi_{n}(u)+o(1),
$$

where

$$
\begin{aligned}
\phi_{n}(u)= & \frac{-a^{n+1}}{n !(n+1) !} \ln (u) \\
& +\sum_{i=n+2}^{\infty} \frac{(-1)^{i+n+1} a^{i} u^{-(i-1-n)}}{i !(i-1)(i-2) \cdots(i-1-n)} .
\end{aligned}
$$


Let $v=a u^{-1}, \phi_{n}(v)$ can be expressed as

$$
\phi_{n}(v)=\frac{-a^{n+1} \ln a}{n !(n+1) !}+a^{n+1} F_{n}(v)
$$

where

$$
F_{n}(v)=\frac{\ln v}{n !(n+1) !}+\sum_{i=n+2}^{\infty} \frac{(-1)^{i+n+1} v^{i-1-n}}{i !(i-1)(i-2) \cdots(i-1-n)} .
$$

Following similar proof steps of Corollary 1 in [15], the limit $\lim _{v \rightarrow+\infty} F_{n}(v)$ can be proved to exist. Hence, by recalling Equation (A.10), $\Phi_{a}$ can be expressed as

$$
\Phi_{a}=\lim _{u \rightarrow 0^{+}} \Phi_{a}(u)=\sum_{n=0}^{\infty}\left[\frac{-a^{n+1} \ln a}{n !(n+1) !}+C_{n} a^{n+1}\right] .
$$

For a sufficiently large $\rho, \Phi_{a}$ can be approximately calculated as $\Phi_{a} \approx-a \ln a$.

\section{Proof of Lemma 5}

It is not difficult to obtain the PDF of $z$ as $f_{z}(z)=$ $M_{r} e^{-z}\left(1-e^{-z}\right)^{M_{r}-1}, z>0$, since $z=\max \left\{z_{1}, \cdots, z_{M_{r}}\right\}$ and $f_{z_{r}}\left(z_{r}\right)=e^{-z_{r}}, r \in\left\{1, \cdots, M_{r}\right\}$, so that

$$
\epsilon\left[e^{-c z}\right]=\int_{0}^{\infty} e^{-c z} f_{z}(z) \mathrm{d} z=M_{r} \int_{0}^{1} t^{c}(1-t)^{M_{r}-1} \mathrm{~d} t .
$$

Using binomial theorem, $\epsilon\left[e^{-c z}\right]$ can be written as

$$
\begin{aligned}
\epsilon\left[e^{-c z}\right] & =M_{r} \sum_{r=0}^{M_{r}-1} \int_{0}^{1} C_{M_{r}-1}^{r}(-1)^{r} t^{c+r} \mathrm{~d} t \\
& =M_{r} \sum_{r=0}^{M_{r}-1} C_{M_{r}-1}^{r} \frac{(-1)^{r}}{c+r+1}
\end{aligned}
$$

where $C_{p}^{q}=\frac{p !}{(p-q) ! q !}, p$ and $q$ are positive integers, and $p \geq$ $q$. Splitting each term on the right side, Equation (A.16) can be calculated as

$$
\begin{aligned}
\frac{\epsilon\left[e^{-c z}\right]}{M_{r}}= & \left(\frac{1}{c+1}-\frac{1}{c+2}\right)+(-1) \cdot \underbrace{\left(M_{r}-2\right)}_{C_{M_{r}-2}^{1}} \\
& \times\left(\frac{1}{c+2}-\frac{1}{c+3}\right)+\cdots+(-1)^{r} \cdot \underbrace{\left(C_{M_{r}-1}^{r}-C_{M_{r}-2}^{r-1}\right)}_{C_{M_{r}-2}^{r}} \\
& \times\left(\frac{1}{c+r+1}-\frac{1}{c+r+2}\right)+\cdots+(-1)^{M_{r}-2} \\
& \times\left(\frac{1}{c+M_{r}-1}-\frac{1}{c+M_{r}}\right) \\
= & \sum_{r=0}^{M_{r}-2} C_{M_{r}-2}^{r} \frac{(-1)^{r}}{(c+r+1)(c+r+2)}
\end{aligned}
$$

Furthermore, by iteratively repeating the similar process in Equation (A.17), the expectation of $e^{-c z}$ can be finally obtained as $\epsilon\left[e^{-c z}\right]=\frac{M_{r} !}{\Pi_{i=1}^{M_{r}}(c+i)}$.

\section{Appendix 2}

\section{Proof of theorem 1}

As shown in [7,22], the ML error probability can be tightly bounded by the outage probability at high SNR, so the outage probability will be analyzed in this section. According to [22] and the protocol description in Section 2, we can define the outage event of the proposed ARDF protocol as $\mathcal{O} \triangleq \bigcup_{k=0}^{2} \mathcal{O}_{A_{k}}$, where $\mathcal{O}_{A_{2}} \triangleq \mathcal{O}_{A_{2}, \bar{E}} \bigcup \mathcal{O}_{A_{2}, E}$. Here, $\mathcal{O}_{A_{k}}$ denotes the outage event in the situation $A_{k}$ at the destination, $\mathcal{O}_{A_{2}, \bar{E}}$ and $\mathcal{O}_{A_{2}, E}$ are similarly defined. Thus, the overall outage probability of the proposed protocol can be expressed as

$$
\begin{aligned}
P(\mathcal{O})= & \sum_{k=0}^{1} P\left(\mathcal{O}_{A_{k}}\right) P\left(A_{k}\right)+P\left(A_{2}, \bar{E}\right) P\left(\mathcal{O}_{A_{2}, \bar{E}}\right) \\
& +P\left(A_{2}, E\right) P\left(\mathcal{O}_{A_{2}, E}\right) \\
\leq & \sum_{k=0}^{1} P\left(\mathcal{O}_{A_{k}}\right) P\left(A_{k}\right)+P(\bar{E}) P\left(\mathcal{O}_{A_{2}, \bar{E}}\right)+P(E) P\left(\mathcal{O}_{A_{2}, E}\right) .
\end{aligned}
$$

The probabilities of each situation $A_{k}$ and the event $E$ have been presented in Lemma 2 and Lemma 3, respectively. Moveover, the outage probability in each situation will be analyzed in the terms of the MAC capacity region. Based on the definition in Equation (1), the source data rate constrains for the proposed-ARDF protocol can be further calculated as follows:

$$
|\mathcal{S}| Q \tilde{R}<\log \left(\operatorname{det}\left(\mathbf{I}+\rho \sum_{l \in \mathcal{L}} \mathbf{h}_{l} \mathbf{h}_{l}^{H}\right)\right), \forall \mathcal{S} \subseteq\{1,2\}
$$

where $|\mathcal{S}|$ denotes number of users in $\mathcal{S}, Q$ denotes the number of codewords transmitted by each source in one cooperative frame, and $\mathbf{h}_{l}$ is a channel vector, both the structure of $\mathbf{h}_{l}$ and the set $\mathcal{L}$ are a function of $\mathcal{S}$ and the details of their relationship to $\mathcal{S}$ will be discussed in the next few subsections. The outage events occur when any constraint in Equation (B.2) is not met, and the highest outage probability achieved by each constraint is the dominant factor [22]. In the following subsections, different values of each parameter in Equation (B.2) will be considered for different situations.

\section{Situation $A_{0}$}

$\mathcal{S} \subseteq\{1,2\}, Q=L$, and $\mathbf{h}_{l}$ denotes the $l$-th column vector of $\mathbf{H}_{A_{0}}$ in Table 1 . When $|\mathcal{S}|=1, \mathcal{S}$ is assumed to be $\{1\}$ without loss of generality, so $\mathcal{L}=\{1,3, \cdots, 2 L-1\}$, and 
the outage probability at the destination in such a case can be calculated as

$$
\begin{aligned}
P_{1}\left(\mathcal{O}_{A_{0}}\right)= & P\left\{\left[1+\rho\left(X_{1}+\alpha_{2}^{2} X_{2}\right)\right]\left[1+\rho\left(\alpha_{1}^{2} X_{1}+\alpha_{2}^{2} X_{2}\right)\right]^{L-1}\right. \\
& \left.<2^{L \tilde{R}}\right\} \stackrel{(a)}{<} P\left\{\left[1+\rho\left(\alpha_{1}^{2} X_{1}+\alpha_{2}^{2} X_{2}\right)\right]^{L}<2^{L \tilde{R}}\right\} \\
& \leq P\left\{\alpha_{1}^{2} X_{1}<\frac{2^{\tilde{R}}-1}{\rho}, \alpha_{2}^{2} X_{2}<\frac{2^{\tilde{R}}-1}{\rho}\right\} \\
& \stackrel{(b)}{\leq} P\left\{X_{1}<\frac{2^{\tilde{R}}-1}{\alpha_{1}^{2} \rho}\right\} P\left\{X_{2}<\frac{2^{\tilde{R}}-1}{\alpha_{2}^{2} \rho}\right\} \\
& \doteq \rho^{-2(1-\tilde{r})},
\end{aligned}
$$

where $X_{i}=\left|h_{S i}\right|^{2}, i=1,2$; (a) holds since $0<\alpha_{1}^{2}<1$, and $(b)$ holds since $X_{1}$ is independent of $X_{2}$. Otherwise, $\mathcal{S}=\{1,2\}$ and $\mathcal{L}=\{1,2, \cdots, 2 L\}$,

$$
P_{2}\left(\mathcal{O}_{A_{0}}\right)=P\left\{\log \left(\operatorname{det}\left(\mathbf{I}_{2 L+1}+\rho \mathbf{H}_{A_{0}} \mathbf{H}_{A_{0}}^{H}\right)\right)<2 L \tilde{R}\right\} .
$$

In order to make the analysis more tractable, a $(2 L+$ $\underset{\sim}{1)} \times(2 L+1)$ square matrix $\widetilde{\mathbf{H}}_{A_{0}}$ is first defined as $\widetilde{\mathbf{H}}_{A_{0}} \triangleq\left[\mathbf{H}_{A_{0}}, \mathbf{0}\right]$, where the zero column vector is $(2 L+1)$ dimensional. It is easy to show that $\mathbf{H}_{A_{0}} \mathbf{H}_{A_{0}}^{H}=\widetilde{\mathbf{H}}_{A_{0}} \widetilde{\mathbf{H}}_{A_{0}}^{H}$ and $\left[\mathbf{I}_{2 L+1}+\rho \widetilde{\mathbf{H}}_{A_{0}} \widetilde{\mathbf{H}}_{A_{0}}^{H}\right]$ is a tridiagonal matrix. According to [30], the determinant of the tridiagonal matrix can be shown iteratively as

$$
D_{n}=\left[1+\rho\left(x_{n}+y_{n-1}\right)\right] D_{n-1}-\rho^{2} x_{n} y_{n-1} D_{n-2} \text {, }
$$

where $D_{n}=\operatorname{det}\left[\mathbf{I}_{2 L+1}+\rho \widetilde{\mathbf{H}}_{n} \widetilde{\mathbf{H}}_{n}^{H}\right]$, and $\widetilde{\mathbf{H}}_{n}$ denotes the $n \times n$ top-left submatrix from $\widetilde{\mathbf{H}}_{A_{0}}, x_{n}$ and $y_{n}$ are the $n$ th element on the principle diagonal and subdiagonal of $\widetilde{\mathbf{H}}_{A_{0}}$, respectively. By using such a property and note that $x_{2 L+1}=0$, the following inequality can be obtained

$$
\begin{aligned}
D_{2 L+1} \geq & \left(1+\rho X_{1}\right)\left(1+\rho \alpha_{1} X_{1}\right)^{L-1}\left(1+\rho \alpha_{1} X_{2}\right)^{L} \\
& +\alpha_{2}^{2 L-1}\left(\rho^{2} X_{1} X_{2}\right)^{L}
\end{aligned}
$$

From Equation (B.5) and Lemma 4, $P_{2}\left(\mathcal{O}_{A_{0}}\right)$ can be upper bounded as

$$
\begin{aligned}
P_{2}\left(\mathcal{O}_{A_{0}}\right) & \leq P\left\{\left(\rho^{2} \alpha_{2}^{2} X_{1} X_{2}\right)^{L}<2^{2 L \tilde{R}}\right\} \\
& \approx-\frac{2^{2 \tilde{R}}}{\alpha_{2}^{2} \rho^{2}} \ln \left(\frac{2^{2 \tilde{R}}}{\alpha_{2}^{2} \rho^{2}}\right) \\
& \doteq \rho^{-2(1-\tilde{r})} .
\end{aligned}
$$

Thus, $P\left(\mathcal{O}_{A_{0}}\right) \dot{\leq} \rho^{-2(1-\tilde{r})}$ can be easily obtained by combining $P_{1}\left(\mathcal{O}_{A_{0}}\right)$ and $P_{2}\left(\mathcal{O}_{A_{0}}\right)$.

\section{Situation $A_{1}$}

$\mathcal{L}=\mathcal{S} \subseteq\{1,2\}, Q=1, \mathbf{h}_{l}$ denotes the $l$-th column vector of $\mathbf{H}_{A_{1}}$ in Table 1 . When $|\mathcal{S}|=1$, assume that $\mathcal{S}=\{1\}$, $P_{1}\left(\mathcal{O}_{A_{1}}\right)$ can be easily obtained as

$$
\begin{aligned}
P_{1}\left(\mathcal{O}_{A_{1}}\right) & =P\left\{X_{1}+\alpha_{2}^{2} X_{2}+G_{1}<\frac{2^{\tilde{R}}-1}{\rho}\right\} \\
& \dot{\leq} \rho^{-2(1-\tilde{r})} \prod_{m=1}^{M_{r}} P\left\{\left|g_{1, m}\right|^{2}<\frac{2^{\tilde{R}}-1}{\rho}\right\} \\
& \doteq \rho^{-\left(M_{r}+2\right)(1-\tilde{r})},
\end{aligned}
$$

where $G_{j}=\left|g_{j, r_{j}}\right|^{2} \triangleq \max \left\{\left|g_{j, 1}\right|^{2}, \cdots,\left|g_{j, M_{r}}\right|^{2}\right\}, j=1,2$. Otherwise, $\mathcal{S}=\{1,2\}, P_{2}\left(\mathcal{O}_{A_{1}}\right)$ is

$$
P_{2}\left(\mathcal{O}_{A_{1}}\right)=P\left\{\log \left(\operatorname{det}\left(\mathbf{I}_{2}+\rho \mathbf{H}_{A_{1}}^{H} \mathbf{H}_{A_{1}}\right)\right)<2 \tilde{R}\right\} .
$$

The determinant of $\left[\mathbf{I}_{2}+\rho \mathbf{H}_{A_{1}}^{H} \mathbf{H}_{A_{1}}\right]$ can be first calculated as

$$
\begin{aligned}
\operatorname{det}\left(\mathbf{I}_{2}+\rho \mathbf{H}_{A_{1}}^{H} \mathbf{H}_{A_{1}}\right)= & \prod_{i=1}^{2}\left[1+\rho\left(X_{1}+G_{1}+\alpha_{i}^{2} X_{2}\right)\right] \\
& -\rho^{2}\left|\alpha_{1} \alpha_{2} X_{2}+g_{M 1}^{*} h_{S 1}\right|^{2}
\end{aligned}
$$

where

$$
\begin{aligned}
\left|\alpha_{1} \alpha_{2} X_{2}+g_{M 1}^{*} h_{S 1}\right|^{2} & \leq\left(\alpha_{1} \alpha_{2} X_{2}\right)^{2}+G_{1} X_{1}+2 \alpha_{1} \alpha_{2} X_{2} \sqrt{G_{1} X_{1}} \\
& \leq\left(\alpha_{1} \alpha_{2} X_{2}\right)^{2}+G_{1} X_{1}+\alpha_{1} \alpha_{2} X_{2}\left(X_{1}+G_{1}\right) .
\end{aligned}
$$

So that det $\left(\mathbf{I}_{2}+\rho \mathbf{H}_{A_{1}}^{H} \mathbf{H}_{A_{1}}\right)>\rho^{2}\left[G_{1}^{2}+\left(1-\alpha_{1} \alpha_{2}\right) X_{1} X_{2}\right]$ can be obtained, where $0<\alpha_{1} \alpha_{2} \leq \frac{1}{2}$, and $P_{2}\left(\mathcal{O}_{A_{1}}\right)$ can be upper bounded as

$$
\begin{aligned}
P_{2}\left(\mathcal{O}_{A_{1}}\right) & \leq P\left\{G_{1}^{2}+\left(1-\alpha_{1} \alpha_{2}\right) X_{1} X_{2}<\frac{2^{2 \tilde{R}}}{\rho^{2}}\right\} \\
& \leq P\left\{X_{1} X_{2}<\frac{2^{2 \tilde{R}}}{\left(1-\alpha_{1} \alpha_{2}\right) \rho^{2}}\right\} P\left\{G_{1}<\frac{2^{\tilde{R}}}{\rho}\right\} \\
& \doteq \rho^{-\left(M_{r}+2\right)(1-\tilde{r})},
\end{aligned}
$$

where the last relationship is based on Lemma 4. Hence, $P\left(\mathcal{O}_{A_{1}}\right) \dot{\leq} \rho^{-\left(M_{r}+2\right)(1-\tilde{r})}$ can be obtained.

\section{Situation $A_{2}$ event $\bar{E}$}

$\mathcal{L}=\mathcal{S} \subseteq\{1,2\}, Q=1, \mathbf{h}_{l}$ denotes the $l$-th column vector of $\mathbf{H}_{A_{2}, \bar{E}}$ in Table 1. When $|\mathcal{S}|=1, P_{1}\left(\mathcal{O}_{A_{2}, \bar{E}}\right) \doteq$ $\rho^{-\left(2 M_{r}+2\right)(1-\tilde{r})}$ can be easily obtained by following the 
similar steps in Equation (B.7). Otherwise, $\mathcal{S}=\{1,2\}$, $P_{2}\left(\mathcal{O}_{A_{2}, \bar{E}}\right)$ can be written as

$$
P_{2}\left(\mathcal{O}_{A_{2}, \bar{E}}\right)=P\left\{\log \left(\operatorname{det}\left(\mathbf{I}_{2}+\rho \mathbf{H}_{A_{2}, \bar{E}}^{H} \mathbf{H}_{A_{2}, \bar{E}}\right)\right)<2 \tilde{R}\right\} .
$$

In the above equation, $\operatorname{det}\left(\mathbf{I}_{2}+\rho \mathbf{H}_{A_{2}, \bar{E}}^{H} \mathbf{H}_{A_{2}, \bar{E}}\right)=K_{1}-$ $K_{2}$, where

$$
\begin{aligned}
K_{1} & =\prod_{i=1}^{2}\left[1+\rho\left(X_{1}+G_{1}+G_{2}+\alpha_{i}^{2} X_{2}\right)\right] \\
& \geq \rho^{2}\left[\left(X_{1}+G_{2}+G_{1}\right)^{2}+X_{2}\left(X_{1}+G_{2}+G_{1}\right)+\alpha_{1} \alpha_{2} X_{2}^{2}\right],
\end{aligned}
$$$$
K_{2}=\rho^{2}\left|\alpha_{1} \alpha_{2} X_{2}+g_{M 1}^{*} h_{S 1}+g_{M 2}^{*} g_{M 1}\right|^{2}
$$$$
\leq \rho^{2}\left[\left(\alpha_{1} \alpha_{2} X_{2}\right)+\sqrt{G_{1}}\left(\sqrt{X_{1}}+\sqrt{G_{2}}\right)\right]^{2}
$$$$
\leq \rho^{2}\left[\left(\alpha_{1} \alpha_{2} X_{2}\right)^{2}+\alpha_{1} \alpha_{2} X_{2}\left(X_{1}+G_{2}+2 G_{1}\right)\right.
$$$$
\left.+2 G_{1}\left(X_{1}+G_{2}\right)\right] \text {, }
$$

so that $\operatorname{det}\left(\mathbf{I}_{2}+\rho \mathbf{H}_{A_{2}, \bar{E}}^{H} \mathbf{H}_{A_{2}, \bar{E}}\right)>\rho^{2}\left[G_{1}^{2}+G_{2}^{2}+\left(1-\alpha_{1}\right.\right.$ $\left.\alpha_{2}\right) X_{1} X_{2}$ ] can be obtained. By using Lemma 4 and following the similar steps in Equation (B.11), $P_{2}\left(\mathcal{O}_{A_{2}, \bar{E}}\right)$ can be upper bounded as

$$
\begin{aligned}
P_{2}\left(\mathcal{O}_{A_{2}, \bar{E}}\right) & \leq P\left\{G_{1}^{2}+G_{2}^{2}+\left(1-\alpha_{1} \alpha_{2}\right) X_{1} X_{2}<\frac{2^{2 \tilde{R}}}{\rho^{2}}\right\} \\
& \doteq \rho^{-\left(2 M_{r}+2\right)(1-\tilde{r})} .
\end{aligned}
$$

Therefore, it can be shown that $P\left(\mathcal{O}_{A_{2}, \bar{E}}\right) \dot{\leq}$ $\rho^{-\left(2 M_{r}+2\right)(1-\tilde{r})}$.

\section{Situation $A_{2}$ event $E$}

The outage probability in situation $A_{2}$ event $E$ is difficult to be obtained using the above method, but the upper bound can be calculated by following the similar analysis in [17]. Firstly, model 4 in Table 1 is assumed to be a symmetric $2 L$-user multiple-access system where the codeword $x(n)$ in $\mathbf{x}_{2 L}$ is transmitted by $\mathrm{S}_{n}$, and such an assumption will make the analysis tractable. For the twouser case considered in this paper, the performance would not be worse than the performance of the former one. So that $\mathcal{L}=\mathcal{S} \subseteq\{1,2, \cdots, 2 L\}, Q=1, \mathbf{h}_{l}$ denotes the $l$-th column vector of $\mathbf{H}$ in Equation (3), and there are $\left(2^{2 L}-1\right)$ source data constrains in Equation (B.2). For each constraint, there exists a probability that the channel condition cannot satisfy it, and the highest outage probability is the dominant factor and achieve the system's DMT.

In order to calculate the outage probability of each constraint, a $(m+3) \times m$ MIMO channel is first considered as $\mathbf{y}_{m}=\mathbf{F}_{m} \mathbf{s}_{m}+\mathbf{w}_{m}$, where $\mathbf{s}_{m} \in \mathcal{C}^{m}, \mathbf{y}_{m}, \mathbf{w}_{m} \in \mathcal{C}^{m+3}$ and $\mathbf{F}_{m}$ is the $(m+3) \times m$ top-left submatrix from $\mathbf{H}$ in Equation (3). According to [17], the outage probability achieved by this system for every $1 \leq m \leq 2 L$ is the same as the highest outage probability for each constraint in Equation (B.2). When $m=1, P_{1}\left(\mathcal{O}_{A_{2}, E}\right) \doteq \rho^{-\left(2 M_{r}+2\right)(1-\tilde{r})}$ can be easily obtained, following the similar steps in Equation (B.7).

When $m>1$, following the similar DMT analysis for the inter-symbol interference (ISI) channel in [31] and the proof steps in [17], the average error probability can be upper bounded by

$$
P_{e} \leq 2(m+3) \cdot \epsilon\left[\exp \left(-c_{1} \bar{\lambda} \rho^{1-\tilde{r}}|\mathbf{f}|^{2}\right)\right],
$$

where $c_{1}$ is a constant, $\mathbf{f}=\left[h_{S 1}, h_{S 2}, g_{1, r_{1}}, g_{2, r_{2}}\right]^{T}, \epsilon[\cdot]$ denotes the expectation of a random variable, and $\bar{\lambda}=$ $\inf _{\mathbf{f} \in \mathcal{C}^{4}} \lambda_{\min }\left(\frac{\mathbf{F}_{m}}{|\mathbf{f}|}\right)>0, \lambda_{\min }(\cdot)$ denotes the minimum singular value of a matrix. By using Lemma $5, P_{e}$ can be upper bounded as

$$
\begin{aligned}
P_{e} & \leq 2(m+3)\left(\frac{1}{c_{1} \bar{\lambda} \rho^{1-\tilde{r}}+1}\right)^{2}\left[\frac{M_{r} !}{\Pi_{i=1}^{M_{r}}\left(c_{1} \bar{\lambda} \rho^{1-\tilde{r}}+i\right)}\right]^{2} \\
& \doteq \rho^{-\left(2 M_{r}+2\right)(1-\tilde{r})} .
\end{aligned}
$$

By observing the fact that $P\left(\mathcal{O}_{A_{2}, E}\right) \dot{\leq} P_{e}$ [7], the outage probability of model 4 in situation $A_{2}$ event $E$ can be upper bounded as $P\left(\mathcal{O}_{A_{2}, E}\right) \dot{\leq} \rho^{-\left(2 M_{r}+2\right)(1-\tilde{r})}$.

Now that recalling Equation (B.1), Lemma 2, and Lemma 3 and considering all the situations, the overall outage probability can be written as

$$
P(\mathcal{O}) \dot{\leq} \rho^{-\min \left\{\left(2 M_{r}+2\right)(1-\tilde{r}),\left(3 M_{r}+1\right)(1-\tilde{r})\right\}} \doteq \rho^{-\left(2 M_{r}+2\right)(1-\tilde{r})} .
$$

Depending on the variable-rate strategy in Equation (34) of [8] and integrating the four transmission modes, the target transmission data rate $R \mathrm{BPCU}$ can be expressed as

$$
R=P\left(A_{0}\right) \frac{(2 L) \tilde{R}}{2 L+1}+P\left(A_{1}\right) \frac{\tilde{R}}{2}+P\left(A_{2}, \bar{E}\right) \frac{2 \tilde{R}}{5}+P\left(A_{2}, E\right) \frac{(2 L) \tilde{R}}{2 L+3} .
$$

One can also refer to [8] to get the mapping criterion from $R$ to $\tilde{R}$. It is not difficult to prove the inequality $P(\bar{E})+$ $P\left(A_{2}\right)-1 \leq P\left(A_{2}, \bar{E}\right) \leq P(\bar{E})$, so $P\left(A_{2}, \bar{E}\right) \doteq P(\bar{E})$ can be obtained in the large-SNR region. From Equation (A.2) and Lemma 3 and substituting $R=r \log \rho, \tilde{R}=\tilde{r} \log \rho$, $P\left(A_{2}, E\right)=P\left(A_{2}\right)-P\left(A_{2}, \bar{E}\right)$ into Equation (B.19), $r$ can be revealed as

$$
\begin{aligned}
r \doteq & \tilde{r}\left\{\frac{2 L}{2 L+1} P_{1}^{2}+P_{1}\left(1-P_{1}\right)+\frac{2}{5} P_{2}\right. \\
& \left.+\frac{2 L}{2 L+3}\left[\left(1-P_{1}\right)^{2}-P_{2}\right]\right\}
\end{aligned}
$$

where $P_{1}=\rho^{-M_{r}(1-\tilde{r})}, P_{2}=\rho^{-\left(M_{r}-1\right)(1-\tilde{r})}$. Following the analysis in Claim 3 of [8] and the similar steps in 
[14], $d(r)=\left(2 M_{r}+2\right)\left(1-\frac{2 L+3}{2 L} r\right)^{+}$can be proved by substituting $\tilde{r}=\frac{2 L+3}{2 L} r$ into Equation (B.18).

\section{Competing interests}

The authors declare that they have no competing interests.

\section{Acknowledgements}

The work of Peng Xu and Xuchu Dai was supported by the National Natural Science Foundation of China (NSFC) under grant number 61471334, the National High Technology Research and Development Program of China (863 Program) under grant number 2012AA01A502, and the National Basic Research Program of China (973 Program: 2013CB329004). The work of Zhiguo Ding was supported by a Marie Curie International Fellowship within the 7th European Community Framework Programme and the UK EPSRC under grant number EP/I037423/1. This paper has been presented in part at IEEE International Conference on Communications (ICC), June, Canada, 2012 [28].

\section{Author details}

${ }^{1}$ Department of Electronic Engineering and Information Science, University of Science and Technology of China, Huangshan Road, P.O.Box No.4, 230027, Hefei, Anhui, China. ${ }^{2}$ School of Computing and Communications, Lancaster University, Bailrigg, Lancaster LA1 4WA, UK. ${ }^{3}$ Department of Electrical and Computer Engineering, University of Cyprus, Kallipoleos 75, Nicosia 1678, Cyprus. ${ }^{4}$ Department of Computer and Telecommunications Engineering, University of Western Macedonia, 2 Eleftherioy Venizeloy street, Kozani, GR 50100, Greece.

\section{Received: 28 December 2013 Accepted: 1 October 2014}

Published: 11 October 2014

\section{References}

1. S Biswas, R Tatchikou, F Dion, Vehicle-to-vehicle wireless communication protocols for enhancing highway traffic safety. IEEE Commun. Mag. 44(1), 74-82 (2006)

2. C-X Wang, X Cheng, $D$ Laurenson, Vehicle-to-vehicle channel modeling and measurements: recent advances and future challenges. IEEE Commun. Mag. 47(11), 96-103 (2009)

3. Z Ding, KK Leung, Cross-layer routing using cooperative transmission in vehicular ad-hoc networks. IEEE J. Selected Areas Commun. 29(3), 571-581 (2011)

4. K Azarian, HE Gamal, P Schniter, On the achievable diversity-multiplexing tradeoff in half-duplex cooperative channels. IEEE Trans. Inf. Theory. 51, 4152-4172 (2005)

5. Z Ding, T Ratnarajah, C Cowan, On the diversity-multiplexing tradeoff for wireless cooperative multiple access systems. IEEE Trans. Signal Process. 55(9), 4627-4638 (2007)

6. EG Larsson, BR Vojcic, Cooperative transmit diversity based on superposition modulation. IEEE Commun. Lett. 9(9), 778-780 (2005)

7. L Zheng, DNC Tse, Diversity and multiplexing : a fundamental tradeoff in multiple antenna channels. IEEE Trans. Inf. Theory. 49, 1073-1096 (2003)

8. JN Laneman, DNC Tse, GW Wornell, Cooperative diversity in wireless networks: efficient protocols and outage behavior. IEEE Trans. Inf. Theory. 50, 3062-3080 (2004)

9. A Bletsas, A Khisti, DP Reed, A Lippman, A simple cooperative diversity method based on network path selection. IEEE J. Select. Areas Comm. 24, 659-672 (2006)

10. RU Nabar, H Bolcskei, FW Kneubuhler, Fading relay channels: performance limits and space-time signal design. IEEE J. Select. Areas in Comm. 22, 1099-1109 (2004)

11. S Yang, J-C Belfiore, Towards the optimal amplify-and-forward cooperative diversity scheme. IEEE Trans. Inf. Theory. 53, 3114-3126 (2007)

12. B Rankov, A Wittneben, Spectral efficient protocols for half-duplex fading relay channels. IEEE J. Select. Areas Commun. 25(2), 379-389 (2007)

13. Y Fan, C Wang, J Thompson, HV Poor, Recovering multiplexing loss through successive relaying using repetition coding. IEEE Trans. Wireless Commun. 6(12), 4484-4493 (2007)

14. CWang, Y Fan, JS Thompson, M Skoglund, HV Poor, Approaching the optimal diversity-multiplexing tradeoff in a four-node cooperative network. IEEE Trans. Wireless Commun. 9(12), 3690-3700 (2010)
15. $P X u, X$ Dai, Z Ding, I Krikidis, K Leung, Approaching miso upper bound: design of new wireless cooperative transmission protocols. IEEE Trans. Wireless Commun. 10(8), 2725-2737 (2011)

16. C Wang, Y Fan, I Krikidis, JS Thompson, HV Poor, Superposition-coded concurrent decode-and-forward relaying, in Proc. IEEE Int. Symp. Inf. Theory (IEEE Toronto, Canada, 2008), pp. 2390-2394

17. CWang, Y Fan, J Thompson, H Poor, A comprehensive study of repetition-coded protocols in multi-user multi-relay networks. IEEE Trans. Wireless Commun. 8(8), 4329-4339 (2009)

18. Z Ding, KK Leung, KK Goeckel, D Towsley, A relay assisted cooperative transmission protocol for wireless multiple access systems. IEEE Trans. Commun. 58(8), 2425-2435 (2010)

19. M Xiao, M Skoglund, Design of network codes for multiple-user multiple-relay wireless networks, in IEEE International Symposium on Inf. Theory (ISIT) (IEEE Seoul, Korea, 2009), pp. 2562-2566

20. CWang, M Xiao, M Skoglund, Diversity-multiplexing tradeoff analysis of coded multi-user relay networks. IEEE Trans. Commun. 59(7), 1995-2005 (2011)

21. CWang, H Farhadi, M Skoglund, Achieving the degrees of freedom of wireless multi-user relay networks. IEEE Trans. Commun. 60(9), 2612-2622 (2012)

22. DNC Tse, $P$ Viswanath, $L$ Zheng, Diversity-multiplexing tradeoff in multiple-access channels. IEEE Trans. Inf. Theory. 50, 1859-1874 (2004)

23. TM Cover, JA Thomas, Elements of Inf. Theory (Wiley, New York, 1991)

24. R Clarke, A statistical theory of mobile-radio reception. Bell Syst. Tech. J. 47(6), 957-1000 (1968)

25. A Akki, Statistical properties of mobile-to-mobile land communication channels. IEEE Trans. Vehicular Technol. 43(4), 826-831 (1994)

26. CY Leow, Z Ding, KK Leung, Linear precoded cooperative transmission protocol for wireless broadcast channels. IEEE Trans. Vehicular Technol. 60(7), 3509-3515 (2011)

27. D Tse, P Viswanath, Fundamentals of Wireless Communication (Cambridge University Press, UK, 2005)

28. P Xu, Z Ding, X Dai, I Krikidis, AV Vasilakos, A novel relay-assisted protocol for cooperative multiple access networks, in IEEE International Conference on Communications (ICC) (IEEE, 2012), pp. 4547-4551

29. IS Gradshteyn, IM Ryzhik, Table of Integrals, Series and Products, 7th edn. (Academic Press, Massachusetts, 2007)

30. GH Golub, CF van Loan, Matrix Computations, 3rd edn. (John Hopkins Univerity Press, Maryland, 1996)

31. LH Grokop, D Tse, Diversity-multiplexing tradeoff in ISI channels. IEEE Trans.Inf. Theory. 55(1), 109-135 (2009)

\section{doi:10.1186/1687-1499-2014-165}

Cite this article as: Xu et al:: Alternative relaying for cooperative multiple-access channels in wireless vehicular networks. EURASIP Journal on Wireless Communications and Networking 2014 2014:165.

\section{Submit your manuscript to a SpringerOpen ${ }^{\circ}$ journal and benefit from:}

- Convenient online submission

- Rigorous peer review

- Immediate publication on acceptance

- Open access: articles freely available online

- High visibility within the field

- Retaining the copyright to your article

Submit your next manuscript at $\boldsymbol{\wedge}$ springeropen.com 